sondern trägt auch zur Profilierung am Markt bei. Außerdem fördert sie deutlich die Qualität des Umweltcontrollings und erhöht die Identifikation der Mitarbeiter mit dem Unternehmen.

- EMAS ist für jene kleinen und mittleren Unternehmen (KMU) geeignet, die über eine ausgeprägte Organisationsstruktur verfügen. Für Kleinstbetriebe mit geringen Umweltrisiken und -wirkungen ist das Kosten-Nutzen-Verhältnis eines formalen Umweltmanagementsystems ungïnstig. Eine Integration weiterer Themen in das Umweltmanagementsystem wie Arbeitssicherheit, Gesundheitsschutz, Hygiene, Produktkennzeichnung und Qualität erhöht die Akzep$\tan z$ der formalen Anforderungen und verbessert das Kosten-Nutzen-Verhältnis.

- KMU benötigen externe Unterstützung für die Umsetzung von EMAS. Insbesondere Kammern, Verbände und zuständige Gewerbeaufsichtsämter müssen ihr Informationsangebot ausbauen und an die Betriebe herantragen.

- Die derzeitige Deregulierungs- und Substitutionsdebatte sollte nicht in erster Linie im Zusammenhang mit EMAS gefiihrt werden, sondern im Zusammenhang mit der generellen Vereinfachung und Systematisierung des Umweltrechts. Das umweltpolitische Instrument EMAS wird ansonsten uiberfrachtet. Außerdem spielt die Deregulierung und Substitution im Zusammenhang mit EMAS aus Sicht der allermeisten teilnehmenden Standorte nur eine untergeordnete Rolle.

\section{Anmerkungen}

(1) Forschungsvorhaben „Evaluation von Umweltmanagementsystemen" im Auftrag des Bundesumweltministeriums/Umweltbundesamtes mit IÖU und ifeu sowie weiteren Unterauftragnehmern( vgl. auch Meldung Seite 28) und "Fachwissenschaftliche Bewertung des EMAS-Systems in Hessen", im Auftrag des Hessischen Ministeriums für Umwelt, Energie, Jugend, Familie und Gesundheit, mit Lahmeyer International, Rechtsanwalt Dr. K. Heuvels und Prof. E. Rehbinder.

(2) Erscheint demnächst als IÖW-Diskussionspapier Nr. $42 / 98$.

\section{Die Autorlinnen}

Das Forschungsfeld Ökologische Unternehmenspolitik des IÖW besteht aus Korthrin Ankele, Jens Clausen, Klaus Fichter, Heinz Kotmann, Thomas Loew und Michael Sleinfeldt.

Kontakt: I0̈W, Giesebrechistr. 13, 10629 Berlin, Tel. 030/88 45 94-0, Fax 030/882 5439 ,

E-mail: mailbox@ioew.b.eunet.de

Entwicklungsperspektiven der ökologischen Leistungsfähigkeit von EMAS

\title{
Ökologischen Nutzen erhöhen
}

\section{Eine Zwischenbilanz der ökologischen Leistungsfähigkeit von EMAS ergibł fol- gendes Bild: Auf der Erfolgsseite können eine gestiegene Kenntnis und Einhal- tung der Umweltgesetze sowie eine verbesserte ökologische Effizienz der Unternehmensstandorte verbucht werden. Angesichts der Konkurrenzsituation zU anderen Umweltmanagementsystemen muB EMAS jedoch zu einem ökologi- schen Gütesiegel weiterentwickelt werden.}

D Von Kathrin Ankele as umweltpolitische Ziel der EG-ÖkoAudit-Verordnung ist es, den betrieblichen Umweltschutz kontinuierlich $\mathrm{zu}$ verbessern. Erreicht werden soll dies durch ein marktwirtschaftliches Instrument, das auf Eigeninitiative der Unternehmen setzt. Eine Zielsetzung, die aufgrund der vielfach konstatierten Grenzen ordnungsrechtlicher Steuerungs- und insbesondere Kontrollmöglichkeiten durchaus sinnvoll erscheint. Alle Aussagen über die Wirksamkeit von EMAS stehen unter dem Vorbehalt, daß die Umsetzung noch in der Anfangsphase steckt. Dennoch kann aufgrund der in Deutschland vergleichsweise regen Beteiligung bereits heute eine Zwischenbilanz gezogen werden, die Anregungen für den Revisionsprozeß auf EUEbene liefert.

Zunächst ist festzuhalten: Die Einfuihrung eines Umweltmanagementsystems ist grundsätzlich positiv $\mathrm{zu}$ beurteilen, da die systematische Beschäftigung mit Umweltschutzfragen fast schon zwangsläufig Optimierungspotentiale offenlegt. Ein weiterer entscheidender Vorteil von EMAS gegenüber anderen Umweltmanagementsystemen ist die Anforderung, betriebliche Umweltschutzziele und -programme aufzustellen und umzusetzen, die explizit der Verringerung von Umweltwirkungen dienen sollen.

Ein nachweisbarer Erfolg der Umsetzung der EGÖko-Audit-Verordnung besteht in der erhöhten Kenntnis und Einhaltung von Umweltgesetzen und -bestimmungen durch die Unternehmen. Dies wirkt sich auch auf deren Umweltschutzleistung aus, da mit zunehmender Gesetzeseinhaltung beispielsweise Grenzwertïberschreitungen vermieden werden. Ferner werden die Stoff- und Energieflüsse des Standortes häufig zum ersten Mal systematisch erhoben und einander in Form von Input-Output-Bilanzen (betrieblichen Ökobilanzen) gegenüber gestellt. Durch deren Ana- lyse und Bewertung werden Schwachstellen entdeckt und beseitigt, was zu standortbezogenen Effizienzsteigerungen und somit zu einer verbesserten Umweltschutzleistung der Unternehmen fiihrt. Im Idealfall wird in Unternehmen durch die EMAS-Teilnahme ein dynamischer Verbesserungsprozeß in Gang gesetzt.

\section{Umweltschutzziele und KVP}

Wesentliche Ansatzpunkte für diese Dynamik von EMAS sind die Anforderungen, Umweltschutzziele und -programme zur Verringerung von Umweltwirkungen umzusetzen und in einem kontinuierlichen Verbesserungsprozeß (KVP) weiterzuentwickeln. Die Praxis zeigt jedoch, daß die Entwicklung von Umweltschutzzielen viele Unternehmen vor Probleme stellt und dieses Potential von EMAS daher unzureichend ausgeschöpft wird. Vor allem fehlt den Unternehmen der Maßstab, verschiedene (konkurrierende) Ziele gegeneinander abzuwägen oder generell mittelfristige Ziele $\mathrm{zu}$ entwickeln. Ferner fehlt bislang der Bezug zu den tatsächlichen Umweltwirkungen. Diese Aspekte sind aber notwendige Voraussetzungen, um Prioritäten für den betrieblichen Umweltschutz setzen zu können. Der Verordnungstext leistet Unternehmen hierbei keine Hilfestellung, da keine materiellen Standards enthalten sind. Letzteres war seitens der Verordnungsgeber nicht beabsichtigt und wird sich im Zuge der Revision wahrscheinlich auch nicht ändern. Es überrascht daher nicht, daß die Unternehmen Umweltschutzziele insbesondere in denjenigen Bereichen formulieren, die gesetzlich geregelt sind oder auch Gegenstand des „klassischen Umweltschutzes“" sind. Dazu zählen Reduzierungen des Abfallaufkommens, der Abwasserbelastung oder des Energieeinsatzes.

In der Anfangsphase der EMAS-Umsetzung mag es ausreichen, Umweltschutzziele in Form von quantitativen Reduktionszielen einzelner Stoff- 
und Energieströme zu formulieren (z.B. zehn Prozent geringeres Sonderabfallaufkommen, fünf Prozent geringerer Energieeinsatz). Daß der betriebliche Umweltschutz auf diese Weise verbessert werden kann, zeigt die bisherige Umsetzungspraxis. Diese Vorgehensweise stößt jedoch an Grenzen, wenn die ersten Reduktionspotentiale ausgeschöpft sind. Unternehmen, die seit langem im Umweltschutz über den gesetzlichen Rahmen hinaus aktiv sind, bestätigen diesen Befund. Der Anspruch des kontinuierlichen Verbesserungsprozesses von EMAS, der auch nicht wenigen Unternehmen Kopfzerbrechen bereitet, verlangt jedoch expressis verbis eine stete Weiterentwicklung. Damit diese Anforderung auch langfristig greift und der Umwelt nützt, sollten Umweltschutzziele für die Bereiche formuliert werden, in denen der größtmögliche Nutzen für die Umwelt mit einem möglichst geringen Aufwand für die Unternehmen verbunden ist. Die Unternehmen sollten somit im eigenen Interesse ihre zeitlichen und finanziellen Ressourcen für den Umweltschutz ökonomisch effizient und ökologisch effektiv einsetzen.

Neben materiellen Zielen wurden durch die Einfuihrung von EMAS auch Ziele formuliert, die sich auf die Organisation des Umweltschutzes oder auf die Sensibilisierung und Qualifikation von Mitarbeitern und Mitarbeiterinnen beziehen. Die ökologischen Wirkungen dieser Ziele und der davon abgeleiteten Maßnahmen können heute noch nicht abschließend beurteilt werden, es steckt jedoch ein erhebliches Potential darin. Denn es ist fast schon Allgemeinwissen, daß Umweltschutz nur dann erfolgreich sein kann, wenn die Belegschaft die Umsetzung trägt.

\section{- Ausgangspunkt Umwelfwirkungen}

Damit die betrieblichen Umweltschutzmaßnahmen $\mathrm{zu}$ einem größtmöglichen ökologischen Nutzen führen, sollten sie künftig am konkreten Handlungsbedarf, d.h. an lokalen, regionalen und globalen Umweltproblemen, ausgerichtet werden, soweit das einzelne Unternehmen zu diesen Problemen beiträgt. Unternehmen müssen sich mit den am Standort hervorgerufenen Umweltwirkungen befassen, um diesen Tatbestand beurteilen zu können.

Die Umsetzungspraxis zeigt jedoch, daß Unternehmen keine klare Vorstellung davon haben, was Umweltwirkungen sind. Darunter sind gerade nicht die Stoffflïsse zu fassen, die in der Praxis fast ausnahmslos untersucht werden.

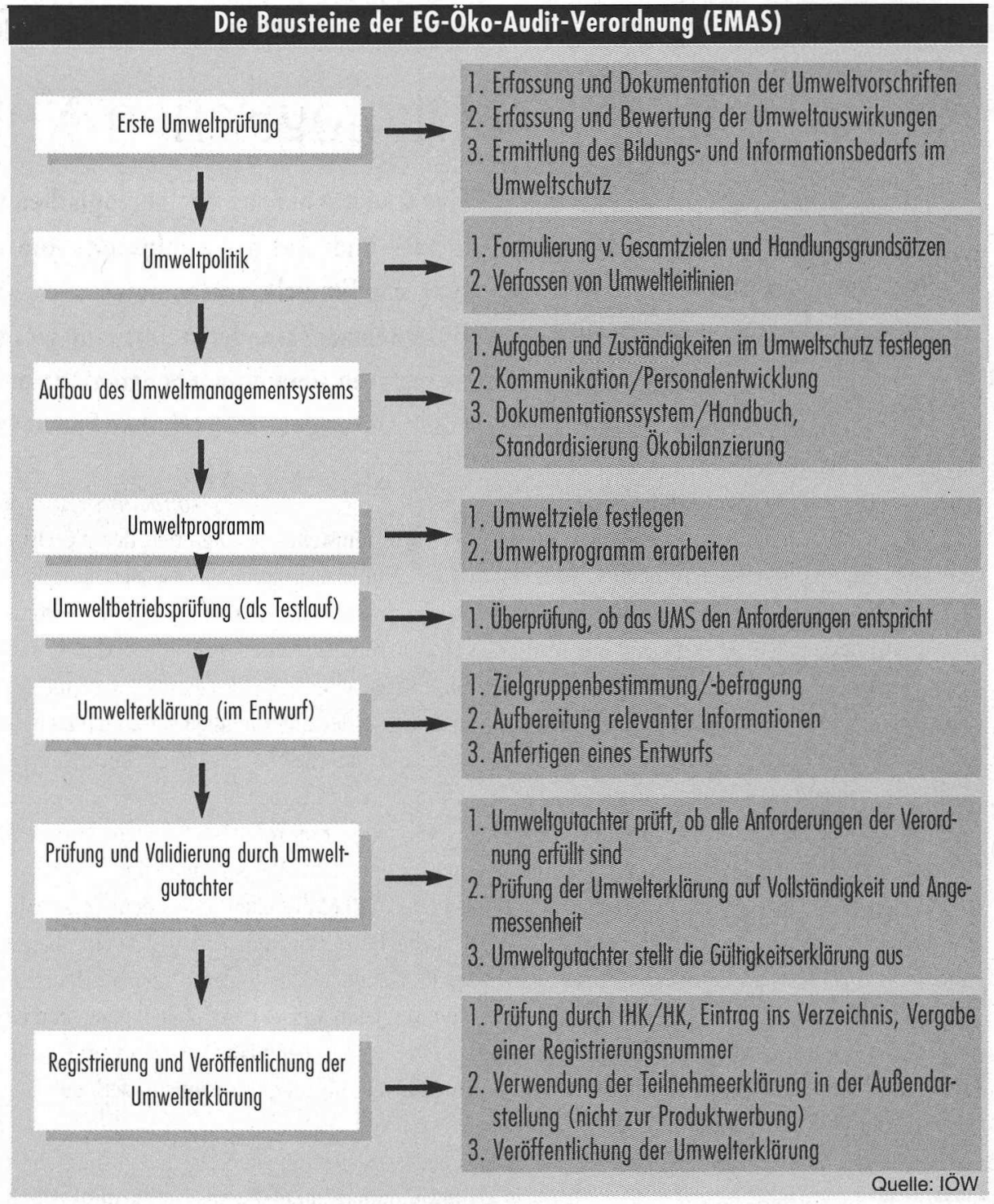

Umweltwirkungen sind dagegen die Wirkungen, die diese Stoffflüsse in der Umwelt hervorrufen können, wie etwa Versauerung oder Eutrophierung. Auch Treibhauseffekt und Ozonabbau fallen darunter und sind trotz der globalen Wirkungszusammenhänge auch auf Standortebene relevant und beeinflußbar.

Damit ein Unternehmen die tatsächlichen Umweltwirkungen der Tätigkeiten ermitteln kann, müssen bestimmte Voraussetzungen erfüllt sein: Informationen über die Umweltsituation am Standort und in der Region sind erforderlich, damit die Prioritäten betrieblicher Umweltschutzmaßnahmen am Handlungsbedarf ausgerichtet werden können. Als Beispiel für Daten über die Umweltsituation können die regelmäßig veröffentlichten „Umweltdaten“ des Landes Baden-Württemberg angeführt werden (1).

Diese Datenbestände müssen jedoch weiterentwickelt werden, damit sie von Unternehmen genutzt werden können. Der Entwicklungsbe- darf besteht einerseits in der räumlichen Auflösung der Informationen, die für ein einzelnes Unternehmen bislang nicht ausreichend genau ist, um Informationen über die Situation am eigenen Standort zu gewinnen. Außerdem sind die Zugriffsmöglichkeiten auf diese Daten für Unternehmen derzeit ungenügend. Die Behörden müssen entsprechend ihren Aufgaben und Kompetenzen die Informationen den Unternehmen aktiv und mit Hilfestellungen zur Interpretation und Auswertung zur Verfügung stellen.

\section{Politische Umweltziele nötig}

Um Prioritäten für betriebliche Umweltschutzmaßnahmen festlegen zu können, ist die Einbeziehung der Umweltsituation am Standort ein erster und notwendiger Schritt. Darüber hinaus sollten jedoch auch (im Idealfall allgemein akzeptierte) politische Umweltziele als Orientierung für den betrieblichen Umweltschutz herangezogen werden. Bislang findet dies in der 
betrieblichen Praxis kaum statt. Umweltqualitätsund Umwelthandlungsziele werden in den letzten Jahren, ausgehend von den Arbeiten der beiden Enquete-Kommissionen „Schutz des Menschen und der Umwelt" des 12. und 13. Deutschen Bundestages, als umweltpolitisches Instrument zunehmend diskutiert und gefordert (2). Ende April veröffentlichte das Bundesumweltministerium den Entwurf eines umweltpolitischen Schwerpunktprogramms ,Nachhaltige Entwicklung in Deutschland“ (3), in dem für prioritäre Handlungsfelder Zielvorgaben sowie Maßnahmen und Akteure dargestellt werden. Die Bedeutung politischer Umweltziele als Orientierung fiur betriebliche Umweltschutzmaßnahmen wird zunehmend erkannt (4). Sie sind eine notwendige Voraussetzung für die Perspektive, EMAS zu einem ökologischen Gütesiegel weiterzuentwickeln.

\section{Die Rolle der Umweltgufachter}

Eine wesentliche Rolle für die Akzeptanz und Glaubwürdigkeit von EMAS spielen die Umweltgutachter. Sie müssen künftig prïfen und beurteilen, ob ein angemessener KVP erreicht wurde und von den Unternehmen für den nächsten Validierungszyklus angestrebt wird. Sie prïfen ebenfalls, ob die Erhebung und Bewertung der Umweltwirkungen ausreichend ist. Um EMAS in der beschriebenen Weise zu einem ökologischen Gütesiegel weiterzuentwickeln, müssen auch die Umweltgutachter die eigentlichen Umweltwirkungen berücksichtigen und als Ausgangspunkt für einen einheitlich geprüften, angemessenen KVP anwenden. Bislang existieren jedoch keine allgemeingültigen inhaltlichen Prüfmaßstäbe für Stoffund Energieflußbilanzen, ökologische Bewertungen oder den KVP. Mit der jüngst in Deutschland durch das Institut der Umweltgutachter veröffentlichten „Richtlinie zum Validierungsverfahren“ wurde nun eine Grundlage zur Vereinheitlichung vorgelegt (5). Darin werden allerdings unter der Rubrik ,Auswirkungen auf die Umwelt“ die in der jetzigen Verordnung aufgeführten Stoff- und Energieflïsse und nicht die tatsächlichen Umweltwirkungen aufgezählt. Es ist zwar auch der in der Praxis bislang unberücksichtigte Anspruch enthalten, zu prïfen ob und wie die ,Auswirkungen auf Teilbereiche der Umwelt und auf Ökosysteme" von den Unternehmen bewertet und registriert werden. Da jedoch in der IdU-Richtlinie eine Auslegung dieser Anforderung fehlt, ist zu vermuten, daß die bisherige Praxis fortgefuihrt wird und dieser Spiegelstrich der Verordnung unberïcksichtigt bleibt. Um das zu vermeiden, sollte eine Anleitung in die Richtlinie aufgenommen werden, wie betriebliche Umweltziele an tatsächlichen Umweltwirkungen und politischen Umweltzielen orientiert werden können und dieser Anspruch somit erfüllt werden kann.

\section{Fazit}

Das Überleben von EMAS hängt auf mittlere Sicht davon $a b$, daß EMAS ein eigenständiges Profil gegenuiber ISO 14001 entwickelt. Die Profilierung kann m. E. nur darin bestehen, daß EMAS zu einem ökologischen Gütesiegel weiterentwickelt wird. Diese Perspektive wird allerdings keineswegs von allen wirtschaftlichen und politischen Akteuren unterstiitzt, und es ist zumindest offen, ob sich diese Forderungen im endguiltigen Verordnungstext wiederfinden. Ansatzpunkt für die Weiterentwicklung von EMAS zu einem ökologischen Gütesiegel ist die Orientierung an den Zielen zur Verringerung von Umweltwirkungen, die EMAS gegenüber anderen Umweltmanagementsystemen auszeichnet. Die weitere Ausgestaltung sollte über eine Verknüpfung betrieblicher Umweltziele mit politischen Umweltzielen erfolgen und auf einer Bewertung der tatsächlichen Umweltwirkungen sowie der Umweltsituation basieren.

\section{Anmerkungen}

(1) Ministerium für Umwelt und Verkehr Baden-Württemberg, Landesanstalt für Umweltschutz: Umweltdaten 95/96, Karlsruhe 1997.

(2) Enquete-Kommission "Schutz des Menschen und der Umwelt“ (Hrsg.): Die Industriegesellschaft gestalten. Perspektiven für einen nachhaltigen Umgang mit Stoffströmen, Bonn 1994.

Enquete-Kommission „Schutz des Menschen und der Umwelt" (Hrsg.): Konzept Nachhaltigkeit. Fundamente für die Gesellschaft von morgen, Zwischenbericht, Bonn 1997.

(3) Bundesumweltministerium: Nachhaltige Entwicklung in Deutschland. Entwurf eines umweltpolitischen Schwerpunktprogramms, Bonn 1998.

(4) Vgl. insbesondere Bundesumweltministerium, Umweltbundesamt: Nachhaltiges Deutschland, Berlin 1997.

(5) Institut der Umweltgutachter und -berater in Deutschland (IdU) e.V.: Richtlinie zum Validierungsverfahren gemäß Verordnung (EWG) Nr. 1836/93, Bonn 1998.

\section{Die Autorin}

Kathrin Ankele ist seit 1993 wissenschaftliche Mitarbeiterin im IÖW im Forschungsfeld Ökologische Unternehmenspolitik.

Kontakt: IÖW, Giesebrechtstr. 13, 10629 Berlin, Tel.: 030/ 8845 94-21, Fax: 030/ 882 54-39, E-mail: mailbox@ioew.b.eunet.de

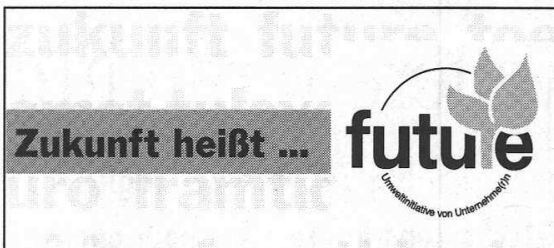

\section{Wenn Sie}

\section{verantwortungsbewuBt wirtschaften wollen ...}

... auf die Entwicklung innovativer und kreativer Konzepte setzen ...

\section{wirtschaftlich handeln müssen ...}

... eine umweltorientierte und sozial verpfilichtete Marktwirtschaft gestalten möchten ...

... europälsch denken können ...

\section{dann machen Sie mit uns}

\section{die Zukunft zur Chance!}




(c) 20I0 Authors; licensee IÖW and oekom verlag. This is an article distributed under the terms of the Creative Commons Attribution Non-Commercial No Derivates License (http://creativecommons.org/licenses/by-nc-nd/3.o/), which permits unrestricted use, distribution, and reproduction in any medium, provided the original work is properly cited. 